\title{
Targeted proteomics of right heart adaptation to pulmonary arterial hypertension
}

\author{
Myriam Amsallem ${ }^{1,2,3,8}$, Andrew J. Sweatt ${ }^{3,4,8}$, Jennifer Arthur Ataam ${ }^{1,2}$, \\ Julien Guihaire ${ }^{5}$, Florence Lecerf ${ }^{5}$, Mélanie Lambert ${ }^{5}$, Maria Rosa Ghigna $\mathbb{1}^{6}$, \\ Md Khadem $\mathrm{Ali}^{3,4}$, Yuqiang $\mathrm{Mao}^{3,4}$, Elie Fadel ${ }^{6}$, Marlene Rabinovitch ${ }^{3,7}$, \\ Vinicio de Jesus Perez ${ }^{3,4}$, Edda Spiekerkoetter ${ }^{3,4}$, Olaf Mercier $^{5}$, \\ Francois Haddad ${ }^{1,2,3,9}$ and Roham T. Zamanian ${ }^{3,4,9}$
}

\begin{abstract}
Affiliations: 'Division of Cardiovascular Medicine, Stanford University School of Medicine, Stanford, CA, USA ${ }^{2}$ Cardiovascular Institute, Stanford University School of Medicine, Stanford, CA, USA. ${ }^{3}$ Vera Moulton Wall Center at Stanford, Stanford University School of Medicine, Stanford, CA, USA. ${ }^{4}$ Division of Pulmonary and Critical Care Medicine, Stanford University School of Medicine, Stanford, CA, USA. ${ }^{5}$ Research and Innovation Laboratory, INSERM U999, Marie Lannelongue Hospital, Paris Sud Saclay University, Le Plessis Robinson, France. ${ }^{6}$ Division of Pathology, Marie Lannelongue Hospital, Le Plessis Robinson, France. ${ }^{7}$ Division of Pediatrics, Stanford University School of Medicine, Stanford, CA, USA. ${ }^{8}$ Both first authors contributed equally. ${ }^{9}$ Both senior authors contributed equally.
\end{abstract}

Correspondence: Myriam Amsallem, Division of Cardiovascular Medicine, Stanford School of Medicine, 300 Pasteur Drive, CA 94305, Stanford, USA. E-mail: myriam.amsallemdgmail.com

@ERSpublications

High plasma HGF levels are associated with right heart maladaptive phenotype and prognosis in PAH. HGF and c-Met RV expression are both increased. Assessing plasma HGF levels might identify patients who warrant closer follow-up and intensified therapy. https://bit.ly/3djiy9O

Cite this article as: Amsallem M, Sweatt AJ, Arthur Ataam J, et al. Targeted proteomics of right heart adaptation to pulmonary arterial hypertension. Eur Respir J 2021; 57: 2002428 [https://doi.org/10.1183/ 13993003.02428-2020].

ABSTRACT No prior proteomic screening study has centred on the right ventricle (RV) in pulmonary arterial hypertension (PAH). This study investigates the circulating proteomic profile associated with right heart maladaptive phenotype (RHMP) in PAH.

Plasma proteomic profiling was performed using multiplex immunoassay in 121 (discovery cohort) and 76 (validation cohort) PAH patients. The association between proteomic markers and RHMP, defined by the Mayo right heart score (combining RV strain, New York Heart Association (NYHA) class and N-terminal pro-brain natriuretic peptide (NT-proBNP)) and Stanford score (RV end-systolic remodelling index, NYHA class and NT-proBNP), was assessed by partial least squares regression. Biomarker expression was measured in RV samples from PAH patients and controls, and pulmonary artery banding (PAB) mice.

High levels of hepatocyte growth factor (HGF), stem cell growth factor- $\beta$, nerve growth factor and stromal derived factor-1 were associated with worse Mayo and Stanford scores independently from pulmonary resistance or pressure in both cohorts (the validation cohort had more severe disease features: lower cardiac index and higher NT-proBNP). In both cohorts, HGF added value to the REVEAL score in the prediction of death, transplant or hospitalisation at 3 years. RV expression levels of HGF and its receptor c-Met were higher in end-stage PAH patients than controls, and in PAB mice than shams.

High plasma HGF levels are associated with RHMP and predictive of 3-year clinical worsening. Both HGF and c-Met RV expression levels are increased in PAH. Assessing plasma HGF levels might identify patients at risk of heart failure who warrant closer follow-up and intensified therapy.

Copyright $\odot$ ERS 2021. This article is open access and distributed under the terms of the Creative Commons Attribution Non-Commercial Licence 4.0. 


\section{Introduction}

Pulmonary arterial hypertension (PAH) is characterised by progressive obliterative vascular remodelling, which leads to increased right heart afterload. Initially, the right ventricle (RV) adapts by increasing its wall thickness (adaptive phenotype), but PAH progression often results in RV enlargement and failure (maladaptive phenotype) with subsequent death or lung transplantation [1]. The pathophysiology underlying this transition from an adapted to maladapted RV is not well elucidated, in part because very few PAH research efforts have focused on establishing markers of the right heart maladaptive phenotype (RHMP). Previous proteomic studies of PAH have uncovered blood profiles associated with pulmonary vascular disease severity and outcomes, including our own work, which utilised unsupervised machine learning to identify PAH phenotypes with distinct inflammatory profiles that stratify clinical risk $[2,3]$. Emerging evidence suggests a link between inflammation and RV failure in PAH patients and experimental disease models $[4,5]$, yet no prior proteomic screening study has centred on the right heart.

Recent imaging studies have substantially improved right heart adaptive phenotyping in PAH, identifying RV free-wall longitudinal strain (RVLS) and end-systolic dimensions (such as the right ventricular end-systolic remodelling index (RVESRI)) as strong echocardiographic markers of RV adaptation $[6,7]$. These markers have each been integrated with New York Heart Association (NYHA) functional class and N-terminal pro-brain natriuretic peptide (NT-proBNP) to define RHMP scores: a Mayo Clinic model (based on RVLS, NYHA class and NT-proBNP) [6] and a Stanford model (RVESRI, NYHA class and NT-proBNP) [7]. The prognostic value of these simple RV-centred models is equivalent to the REVEAL score [7], a widely adopted PAH risk stratification tool, which requires more extensive clinical data and invasive haemodynamic measures.

Building upon these right heart scores, our primary objective was to identify novel circulating proteomic biomarkers associated with RHMP in PAH. We hypothesised that PAH patients may express a specific blood proteomic profile in association with RHMP, independent of pulmonary disease severity. In addition, we aimed to examine the relationship of these proteomic markers to survival. Finally, we sought to assess tissue-level expression of the RHMP markers in RV samples from end-stage PAH patients and an experimental mouse model of RV pressure overload, to test the hypothesis that increased circulating levels reflect RV overexpression, and to also validate the pulmonary artery banding (PAB) model for future mechanistic studies.

\section{Material and methods \\ Study population}

This prospective study included group 1 PAH patients enrolled into the Stanford University Pulmonary Hypertension Biobank (Stanford, CA, USA) between 2008 and 2014, who had plasma collected for proteomic profiling within 2 weeks of a routine echocardiogram (figure 1 and supplementary material). $\mathrm{PAH}$ diagnosis required mean pulmonary arterial pressure $(\mathrm{mPAP}) \geqslant 25 \mathrm{mmHg}$, pulmonary arterial wedge pressure $\leqslant 15 \mathrm{mmHg}$ and pulmonary vascular resistance $(\mathrm{PVR})>240 \mathrm{dyn} \cdot \mathrm{s} \cdot \mathrm{cm}^{-5}$. Exclusion criteria were chronic infection $(n=7)$, primary immunodeficiency $(n=1)$, recent acute illness other than heart failure (within 1 month) $(n=8)$, congenital systemic-to-pulmonary shunt $(n=10)$, active malignancy $(n=4)$, missing echocardiogram within 2 weeks $(n=68)$ or suboptimal echocardiographic data $(n=6)$. Study baseline was the date of blood collection. Patients were divided into a discovery cohort ( $\mathrm{n}=121,2008-$ 2011) and a validation cohort $(n=76,2012-2014)$. In addition, plasma was obtained from healthy controls $(\mathrm{n}=88)$, who underwent rigorous screening to establish health. Stanford institutional review board (IRB) approved the study (IRB \#14083 and \#20942); all subjects provided written informed consent.

\section{Blood proteomic profiling}

Fasting blood samples were collected from an antecubital vein, processed to plasma and stored according to protocols (supplementary material). A targeted proteomic panel of 48 cytokines, chemokines and growth factors (table 1 and supplementary table E1) was measured for each subject using Bio-Plex multiplex immunoassay (BioRad, Hercules, CA, USA), a bead-based flow cytometric platform built on Luminex xMAP technology (Austin, TX, USA), according to manufacturer's instructions (supplementary material). Each sample was measured in duplicate. A Luminex 200 plate reader quantified median fluorescence intensity (MFI) for each protein. MFI was used for all analyses rather than standard curve-derived absolute concentrations, as MFI does not require detection limit censoring and has better

This article has an editorial commentary: https://doi.org/10.1183/13993003.04506-2020

This article has supplementary material available from erj.ersjournals.com

Received: 20 June 2020 | Accepted: 6 Oct 2020 
Clinical circulating proteomics profiling of right heart maladaptive phenotypes in $\mathrm{PAH}$

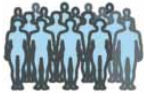

\begin{tabular}{|l|l|} 
Discovery cohort $(\mathrm{n}=121)$ & Validation cohort $(\mathrm{n}=76)$ \\
\hline PLS regression
\end{tabular}

Healthy cohort ( $n=88)$

Identification of circulating RHMP biomarkers

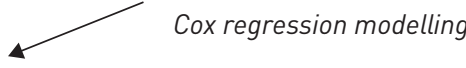

Correlation with 3-year clinical worsening (death, lung transplant or readmission), incremental value over risk scores (REVEAL)

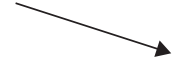

Correlation with right heart remodelling improvement at 1 year in survivors

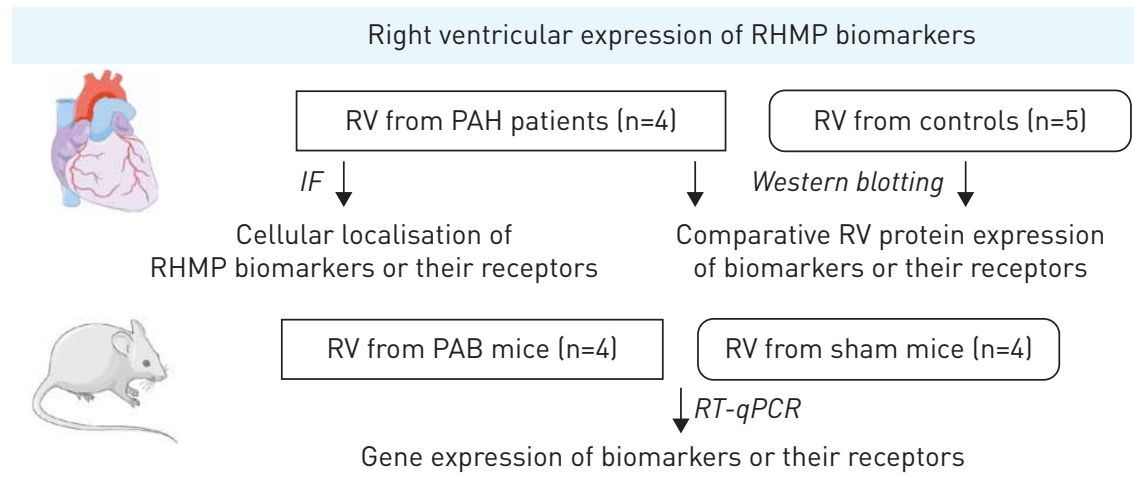

FIGURE 1 Study design. PAH: pulmonary artery hypertension; PLS: partial least squares; RHMP: right heart maladaptive phenotype; RV: right ventricle; IF: immunofluorescence; PAB: pulmonary arterial banding; RT-qPCR: reverse transcriptase quantitative PCR.

downstream statistical power [8]. The inter-assay coefficient of variation was $<15 \%$ for all measured analytes, based on internal controls included across runs.

\section{Clinical data collection}

Transthoracic echocardiogram studies were acquired using Philips IE 33 ultrasound systems (Amsterdam, the Netherlands). RV dimensions and functional metrics were measured on RV-focused apical four-chamber views, averaged over three cycles, and included RVESRI (defined by the lateral wall to septal height ratio) and RVLS, as published previously (supplementary material) [7]. These measurements were used to calculate two right heart adaptive phenotyping scores, the Mayo Clinic score integrating RVLS, NYHA class and NT-proBNP, and the Stanford score integrating RVESRI, NYHA class and NT-proBNP (supplementary table E2). Follow-up echocardiograms available in survivors at 1 year were interpreted using the same methodology. Right heart remodelling with therapy was defined by changes in RVESRI from baseline (improvement if relative change $<10 \%$, worsening if change $>10 \%$, and stable otherwise), as published previously [7].

\section{TABLE 1 Circulating proteomic biomarkers assessed in the study using flow cytometry multiplex arrays}

Biomarkers

IL

IL receptors

Chemokines

Growth factors Other cytokines
IL-1 $\alpha$, IL-1 $\beta$, IL-2, IL-3, IL-4, IL-5, IL-6, LIF, IL-7, IL-9, IL-10, IL-12p70, IL-13, IL-15, IL-16, IL-17, IL-18 IL-1R $\alpha$, IL-2R $\alpha$

$M C P 1=C C L 2, M I P 1 \alpha=C C L 3, M I P 1 \beta=C C L 4$, RANTES=CCL5, MCP3=CCL7, CTACK=CCL27, eotaxin, GROa=CXCL1, IL-8=CXCL8, $M I G=C X C L 9, I P 10=C X C L 10, S D F 1=C X C L 12$

MCSF=CSF1, NGF, SCF, SCGF $\beta$, TNF- $\alpha$, TNF- $\beta 1$, HGF, PDGFbb, FGF2=FGF- $\beta$, GCSF, GMCSF, VEGF-A MIF, TRAIL, IFNA2, IFN- $\gamma$

IL: interleukin; LIF: leukaemia inhibitory factor; SDF: stromal cell-derived factor; MCSF: macrophage colony-stimulating factor; CSF: colonystimulating factor; NGF: nerve growth factor; SCF: stem cell factor; SCGF: stem cell growth factor; TNF: tumour necrosis factor; HGF: hepatocyte growth factor; PDGF: platelet-derived growth factor; FGF: basic fibroblast growth factor; GCSF: granulocyte colony-stimulating factor; GMCSF: granulocyte-macrophage colony-stimulating factor; VEGF: vascular endothelial growth factor; MIF: macrophage migration inhibitory factor; TRAIL: TNF-related apoptosis-inducing ligand; IFNA2: interferon $\alpha 2$; IFN: interferon. 
NYHA class, 6-min walk distance, diffusing capacity of the lung for carbon monoxide, Modification of Diet in Renal Disease estimated glomerular filtration rate and NT-proBNP levels were collected if available within 1 month of baseline. Haemodynamic data (right atrial pressure, mPAP, pulmonary arterial wedge pressure, PVR and cardiac index) were obtained when right heart catheterisation was performed within 3 months of inclusion. Data were used to calculate the REVEAL risk score (supplementary table E3) [9].

Patients were prospectively followed to evaluate the primary endpoint of clinical worsening (i.e. death, lung transplantation or hospitalisation for acute right heart failure), and the secondary end-point (death or lung transplant).

\section{Pathology analysis of human RV samples}

RV frozen and paraffin-embedded biopsies of explanted hearts were obtained from four PAH patients (supplementary table E4) who underwent heart-lung transplant at Marie Lannelongue Hospital (IRB \#18.06.06; Le Plessis-Robinson, France). For comparison, we acquired RV frozen biopsies from five control heart donors (without cardiovascular disease or pulmonary hypertension $(\mathrm{PH})$ ) whose organs were not transplanted (paraffin-embedded biopsies were not available). Explanted hearts were procured from the INSERM URMS 1148 biobank (BRIF BB-0033-00029, BBMRI-EU/infrastructure), approved by the French National Ethics Committee (PFS17-002) and declared at the French Ministry of Research (DC-2018-3141).

Upon identifying RHMP circulating biomarkers in the clinical cohorts, we 1) compared tissue-level expression of these proteins and their receptors in $\mathrm{PAH}$ and controls by incubating frozen RV samples with primary specific antibodies (Abcam, Cambridge, UK) and performing Western blots; and 2) localised the RHMP biomarkers in RV tissue from PAH patients with immunofluorescence staining and confocal microscopy (Zeiss LSM 800 microscope with ZEN software; Carl Zeiss Microscopy, White Plains, NY, USA) (supplementary material).

\section{PAB mouse model RV analysis}

All animal experiments were performed in accordance with National Research Council guidelines [10] and approved by local authorities (Administrative Panel on Laboratory Animal Care, Stanford University, protocol \#27626). Chronic RV pressure overload was induced in 10-14-week-old male C57BL/6 mice as previously published [11] and detailed in the supplementary material. Anaesthetised animals underwent thoracotomy; 6-0 silk sutures were used to band the main pulmonary artery (PA); and 1 week post-surgery a peak pressure gradient $>15 \mathrm{mmHg}$ was confirmed across the band by echocardiography (GE Vivid 7; General Electric Healthcare, Chicago, IL, USA). A control group of age-matched littermates underwent sham surgery (PA isolation without suture placement). mPAP, heart rate and pulmonary valve velocity time integral were measured by echocardiography after 5 weeks to document reduced cardiac output, and RV samples were collected and snap frozen. After isolating RNA from homogenised RV samples, we applied quantitative reverse transcriptase PCR (Applied Biosystems, Foster City, CA, USA) to measure expression levels of the mRNA transcripts corresponding to RHMP proteomic markers of interest.

\section{Statistical analysis}

Baseline characteristics were compared using t-test or Mann-Whitney-Wilcoxon test for continuous data, and Chi-squared test for categorical data. Proteomic data pre-processing involved background fluorescence subtraction, plate/batch adjustment (empirical Bayes methodology) [12], robust quantile normalisation, duplicate averaging and adjustment for age, sex and body mass index. To identify RHMP markers, partial least squares (PLS) regression models were fit using the "SIMPLS" algorithm (supplementary material). RHMP markers were selected by assessing 1) variable importance for projections scores and 2) regression $\beta$-coefficients (using t-tests of coefficients based on variance estimates from jack-knife resampling). PLS models were fit to associate proteins with Mayo and Stanford scores, individual components of each score and pulmonary haemodynamics. RHMP biomarkers were identified as the common biomarkers associated with RHMP in both cohorts. Levels of the identified RHMP biomarkers were examined among low, intermediate and high-risk RV phenotypes (based on Mayo and Stanford scores) to compare each risk group to controls (Kruskal-Wallis and post hoc Dunn tests) and confirm a trend across ordered PAH groups (Cuzick test). To relate biomarkers to outcomes, Kaplan-Meier estimates of transplant- and hospitalisation-free survival were compared across quartiles by log-rank statistics, and Cox proportional hazards regression models were fit. Circulating biomarkers were added as continuous variables. Scaled Schoenfeld residuals were plotted against time for each variable, to ensure the proportional hazards assumption was met. Hazard ratios were normalised to the standard deviation of predictor variables. To determine whether each biomarker added incremental value to established risk scores for prediction of 3-year outcome, Chi-squared values were compared. Binary logistic regression model was used to determine whether biomarkers or right heart metrics were predictive of RVESRI improvement (relative change $<10 \%)$ at 1 year. Patients included in 2014 with $<3$ years' follow-up $(n=4)$ were right censored. 
Statistical analyses were performed using SPSS (version 19; SPSS, Chicago, IL, USA) and R (version 3.5.1; R Core Team, Vienna, Austria) software programmes.

\section{Results}

Proteomic profiling of RHMP in patients with PAH

Table 2 summarises baseline characteristics of the discovery cohort. High levels of hepatocyte growth factor (HGF), stem cell growth factor (SCGF $\beta$ or CLEC11A), nerve growth factor (NGF) and stromal cell-derived factor 1 (SDF1 or CXCL12) were more strongly associated with higher-risk Mayo and Stanford scores, RV dysfunction (RVLS), RV adverse remodelling (RVESRI), higher NT-proBNP and worse NYHA functional class than with pulmonary disease severity (figure 2a; supplementary table E5 and figure E1).

The validation cohort had more severe pulmonary disease features, fewer patients on background therapy and a higher frequency of incident cases than the discovery cohort (table 2). REVEAL, Mayo and Stanford risk score distributions were similar in the two cohorts. High levels of HGF, SCGF $\beta$, NGF, and, to a lesser extent SDF1, were associated with higher-risk Mayo and Stanford scores, RV dysfunction, higher NT-proBNP and worse NYHA functional class (figure 2a). No measured cytokine was found to have a positive association with favourable right heart metrics across both cohorts.

Figure $2 \mathrm{~b}$ compares the four candidate RHMP markers levels in both cohorts combined among low-, intermediate- and high-risk RV patient subgroups, and the 88 healthy controls (median age 58 years, interquartile range (IQR) 44-64 years; 52.3\% were female). Circulating levels of the four markers did not differ according to sex in patients or in controls (supplementary figure E2). HGF and NGF levels did not significantly differ according to ethnicity and race among patients with PAH (supplementary figure E2), while non-Hispanic White patients had lower levels of SCGF $\beta$ than Hispanic patients $(\mathrm{p}<0.01)$ and lower SDF1 $\alpha$ levels than Hispanic and Asian patients $(\mathrm{p}=0.04)$.

\section{Prognostic value of HGF}

During a median follow-up of $3.14(2.16-5.44)$ years, the primary end-point was reached in 76 patients (58 were hospitalised, 10 transplanted and overall 41 died). Event-free survival rates $( \pm \mathrm{SE}$ ) for the primary end-point were $91 \pm 2 \%$ at 1 year, $78 \pm 3 \%$ at 3 years, and $62 \pm 34 \%$ at 5 years (figure $3 a$ ). The secondary end-point of death or transplant was reached in 49 patients.

Using univariable analysis, HGF and SCGF $\beta$ were associated with the primary end-point (hazard ratio (HR) 1.54, 95\% CI 1.24-1.91; $\mathrm{p}<0.0001$, and 1.26, 1.12-1.59; $\mathrm{p}=0.02$, respectively), while NGF $(\mathrm{p}=0.83$ ) and SDF1 $\alpha(\mathrm{p}=0.89)$ were not. Kaplan-Meier survival curves for the primary end-point according to HGF $(\mathrm{p}<0.001)$ and SCGF $\beta$ quartiles $(\mathrm{p}=0.002)$ are presented in figure $3 \mathrm{~b}$ and supplementary figure E3. HGF added significant incremental value to the Mayo, Stanford and REVEAL scores for prediction of the primary end-point at 3 years $(p<0.05$; figure $3 c$ and supplementary table E6). Both HGF and SCGF $\beta$ were also associated with the secondary end-point of death or transplant (HR 1.71, 95\% CI 1.38-2.12; $\mathrm{p}<0.0001$, and $1.26,1.01-1.59 ; \mathrm{p}=0.02$, respectively).

\section{Absence of biomarker of longitudinal cardiac remodelling}

Among the total population, 174 patients (93.0\% of 1-year survivors) had a follow-up echocardiogram at 1 year (median (IQR) time to follow-up study was 1.03 (0.85-1.35) years). Parenteral prostanoids were initiated in 37 patients between baseline and follow-up, while $\mathrm{PH}$-specific therapies were not changed in 55 patients. None of the four RHMP biomarkers at baseline (entered as continuous variables using binary regression analyses) were found to be significantly associated with changes in right heart remodelling, irrespective of the therapy initiated between baseline and the follow-up echocardiogram. Similarly, the Stanford score $(\mathrm{p}=0.50)$, Mayo score $(\mathrm{p}=0.99)$ and NT-proBNP (OR 1.85, 95\% CI 0.98-3.49; $\mathrm{p}=0.06$ ) at baseline were not associated with changes in right heart remodelling at follow-up.

\section{Human RV tissue-level expression of RHMP biomarkers}

Protein levels of HGF and its receptor c-Met were higher in RV specimens from PAH patients than controls (figure 4). The levels of NGF were decreased and its receptor tropomyosin-related receptor kinase A (TrkA) increased in PAH as compared to controls. In patients with PAH, RV fibrosis level assessed by the percentage area of picro-sirius red staining was $11.5 \%(8.8-15.7 \%)$, similar across PAH aetiology (supplementary figure E4 and supplementary material). Figure 5 and supplementary figure E5 show the localisation of the four biomarkers and receptors in RV samples from the patient with idiopathic PAH. Both PAH patients (idiopathic and bone morphogenetic protein receptor (BMPR)2 mutation) exhibited qualitatively similar protein RV expression. The receptor c-Met colocalised with cardiomyocytes, smooth muscle cells, endothelial cells and fibroblasts markers, and to a lesser extent with macrophages (figure 5). 
TABLE 2 Comparative baseline characteristics of the discovery and validation cohorts

\begin{tabular}{|c|c|c|c|}
\hline & Discovery cohort & Validation cohort & p-value \\
\hline Subjects & 121 & 76 & \\
\hline Age years & $50(39-59)$ & $52(43-61)$ & 0.20 \\
\hline Female & $90(74.4)$ & $66(86.8)$ & 0.06 \\
\hline Race, ethnicity & & & 0.06 \\
\hline White, non-Hispanic & $72(59.5)$ & $41(53.9)$ & \\
\hline Hispanic & $20(16.5)$ & 15 (19.7) & \\
\hline Asian & $22(18.2)$ & 7 (9.2) & \\
\hline Black, non-Hispanic & 4 (3.3) & 6 (7.9) & \\
\hline Other & $3(2.5)$ & $7(9.2)$ & \\
\hline Body mass index $\mathrm{kg} \cdot \mathrm{m}^{-2}$ & $26.9(22.5-31.9)$ & $27.4(24.7-33.5)$ & 0.12 \\
\hline PAH aetiology & & & 0.40 \\
\hline Connective tissue disease & $40(33.1)$ & $31(40.8)$ & \\
\hline Idiopathic & $34(28.1)$ & $14(18.4)$ & \\
\hline Drugs and toxins & $21(17.4)$ & $18(23.7)$ & \\
\hline Congenital heart disease & $13(10.7)$ & 7 (9.2) & \\
\hline Portal pulmonary hypertension & $11(9.1)$ & $5(6.6)$ & \\
\hline Heritable PAH & $2(1.7)$ & $1(1.3)$ & \\
\hline NYHA functional class & & & 0.26 \\
\hline 1 & $8(6.6)$ & $5(6.6)$ & \\
\hline ॥ & 48 (39.7) & $20(26.3)$ & \\
\hline III & $50(41.3)$ & $41(53.9)$ & \\
\hline IV & $15(12.4)$ & $10(13.2)$ & \\
\hline 6MWD m & $427(342-537)$ & $392(299-476)$ & 0.03 \\
\hline NT-proBNP pg.mL ${ }^{-1}$ & 235 (67-977) & $536(150-1441)$ & 0.04 \\
\hline$D_{\text {Lco }} \%$ pred & $73(60-86)$ & $69(53-92)$ & 0.91 \\
\hline MDRD estimated glomerular filtration rate $\mathrm{mL} \cdot \mathrm{min}^{-1} \cdot 1.73 \mathrm{~m}^{-2}$ & $68(53-90)$ & $66(54-79)$ & 0.21 \\
\hline \multicolumn{4}{|l|}{ Haemodynamics } \\
\hline Right atrial pressure $\mathrm{mmHg}$ & $7(5-11)$ & $7(5-12)$ & 0.49 \\
\hline Mean pulmonary arterial pressure $\mathrm{mmHg}$ & $50(40-59)$ & $49(40-58)$ & 0.78 \\
\hline Pulmonary arterial wedge pressure $\mathrm{mmHg}$ & $10(8-13)$ & $10(7-14)$ & 0.72 \\
\hline Pulmonary vascular resistance Wood units & $9.9(6.1-14.2)$ & $10.3(6.8-15.7)$ & 0.56 \\
\hline Cardiac index $\mathrm{L} \cdot \mathrm{min}^{-1} \cdot \mathrm{m}^{-2}$ & $2.2(1.8-2.5)$ & $2.0(1.6-2.3)$ & 0.03 \\
\hline \multicolumn{4}{|l|}{ Echocardiographic metrics } \\
\hline RVESAI $\mathrm{cm}^{2} \cdot \mathrm{m}^{-2}$ & $12.7(10.5-16.4)$ & $12.9(10.2-16.2)$ & 0.81 \\
\hline RVESRI & $1.4(1.3-1.6)$ & $1.5(1.3-1.6)$ & 0.43 \\
\hline RVLS absolute \% & $16.9(13.9-20.0)$ & $16.6(13.2-21.1)$ & 0.93 \\
\hline RVFAC $\%$ & $27.1(22.7-30.7)$ & $26.6(23.1-32.8)$ & 0.91 \\
\hline TAPSE cm & $1.8(1.4-2.1)$ & $1.7(1.3-2.0)$ & 0.20 \\
\hline \multicolumn{4}{|l|}{ PAH therapy } \\
\hline Therapy extent & & & $<0.01$ \\
\hline Treatment naïve & 35 (28.9) & $38(50.0)$ & \\
\hline Monotherapy & $34(28.1)$ & $24(31.6)$ & \\
\hline Dual therapy & $36(29.8)$ & $12(15.8)$ & \\
\hline Triple therapy & $16(13.2)$ & $2(2.6)$ & \\
\hline Phosphodiesterase- 5 inhibitors & $62(51.2)$ & $28(36.8)$ & 0.04 \\
\hline Endothelin receptor antagonists & $39(32.2)$ & $12(15.8)$ & 0.01 \\
\hline Prostanoid therapy & 53 (43.8) & $14(18.4)$ & $<0.01$ \\
\hline REVEAL risk score & & & 0.48 \\
\hline Low & 74 (61.2) & $46(60.5)$ & \\
\hline Average/moderately high & $17(14.0)$ & 15 (19.7) & \\
\hline High/very high & 30 (24.8) & 15 (19.7) & \\
\hline Mayo right heart score & & & 0.85 \\
\hline Low score (0-5) & 50 (41.3) & 33 (43.3) & \\
\hline Intermediate score (6-8) & $40(33.1)$ & $28(36.8)$ & \\
\hline High score $(>9)$ & $24(19.8)$ & $15(19.7)$ & \\
\hline Stanford right heart score & & & 0.72 \\
\hline Low score $(0-2)$ & 49 (40.5) & $30(39.5)$ & \\
\hline Intermediate score (3) & $46(38.0)$ & 26 (34.2) & \\
\hline High score $(>4)$ & 26 (21.5) & $20(26.3)$ & \\
\hline
\end{tabular}

Values are expressed as $n$, median (interquartile range) or $n(\%)$, unless otherwise stated. Significant $p$-values $(<0.05)$ are presented in bold. Right ventricular (RV) systolic pressure was estimated from the tricuspid regurgitation signal in 194 (85\%) patients. PAH: pulmonary arterial hypertension; NYHA: New York Heart Association; 6MWD: 6-min walk distance; NT-proBNP: N-terminal pro-brain natriuretic peptide; $D_{\text {Lco: }}$ diffusing capacity of the lung for carbon monoxide; MDRD: Modification of Diet in Renal Disease; RVESAl: RV end-systolic area indexed on body surface area; RVESRI: RV end-systolic remodelling index; RVLS: RV free-wall longitudinal strain; RVFAC: RV fractional area change; TAPSE: tricuspid annular plane systolic excursion. 


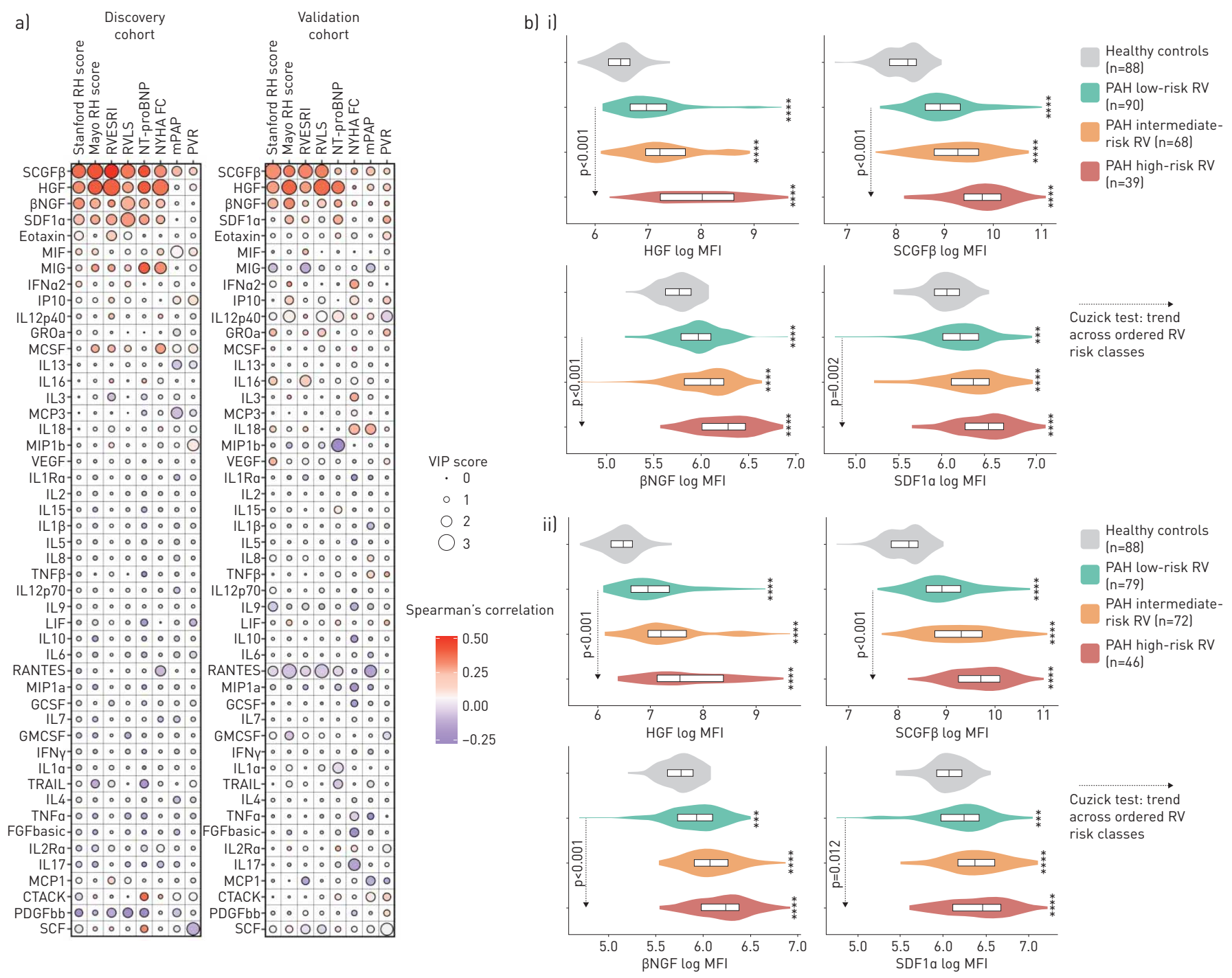

FIGURE 2 a) Variable importance in projection (VIP) scores and correlations for each cytokine with respect to right heart scores and metrics in both cohorts and b) levels of the four markers according to levels of right heart (RH) maladaptive phenotype in patients with pulmonary arterial hypertension (PAH) and controls. a) Using partial least squares regression, we assessed the correlations between plasma proteomic markers (using multiplex immunoassay) and markers of right heart maladaptive phenotype (Stanford RH score based on right ventricular (RV) end-systolic remodelling index (RVESRI), New York Heart Association functional class (NYHA FC) and N-terminal pro-brain natriuretic peptide (NT-proBNP) levels; Mayo RH score based on RV free-wall longitudinal strain (RVLS), NYHA FC and NT-proBNP levels; RVESRI; RVLS; NT-proBNP; NYHA FC; mean pulmonary artery pressure (mPAP) and pulmonary vascular resistance (PVR)) in 121 (discovery cohort, from 2008 to 2011 ) and 76 (validation cohort, from 2012 to 2014) PAH patients. Positive correlations are shown in red, negative correlations in blue. RVLS is presented in absolute value (lowest values indicate worst RV dysfunction). b) Analyte levels of the four strongest biomarkers associated with right heart maladaptive phenotype according to tertiles of the i) Mayo RH score or ii) Stanford RH score in patients with PAH and in the 88 healthy controls. Comparison between each right heart risk category to controls was performed using Dunn's and Kruskal-Wallis test lacross-groups among patients with PAH) with post hoc pairwise comparisons adjusted for multiple testing (Benjamini-Hochberg). ${ }^{* *}: p<0.01 ;{ }^{* * *}: p<0.001 ;{ }^{* * * *}$ : $p<0.0001$. The $p$-values of the Cuzick test were used to confirm an increasing trend of cytokine levels across ordered categories (from low to intermediate to high). SCGF: stem cell growth factor; HGF: hepatocyte growth factor; NGF: nerve growth factor; SDF: stromal cell-derived factor; MIF: macrophage migration inhibitory factor; IFN: interferon; IL: interleukin; MCSF: macrophage colony-stimulating factor; VEGF: vascular endothelial growth factor; TNF: tumour necrosis factor; LIF: leukaemia inhibitory factor; GCSF: granulocyte colony-stimulating factor; GMCSF: granulocyte-macrophage colony-stimulating factor; TRAIL: TNF-related apoptosis-inducing ligand; FGF: fibroblast growth factor; PDGF: platelet-derived growth factor; SCF: stem cell factor.

Punctiform expression of HGF was observed in a vesicle pattern, more abundantly in cardiomyocytes, smooth muscle cells and to a lesser extent in macrophages and fibroblasts. Both TrkA and NGF colocalised with cardiomyocytes, smooth muscle cells and macrophages, while TrkA additionally colocalised with fibroblasts and NGF with endothelial cells (supplementary figure E5). SCGF $\beta$ was present in cardiomyocytes, smooth muscle cells and fibroblasts, and SDF1 was found in cardiomyocytes, smooth muscle cells, endothelial cells and macrophages. 
a)

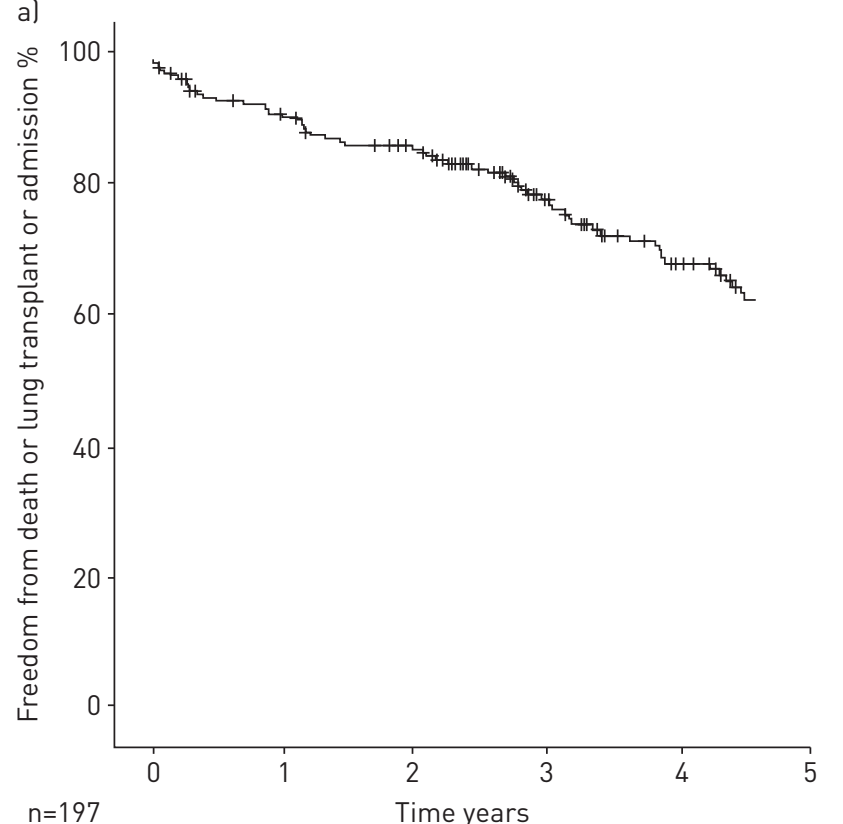

b)

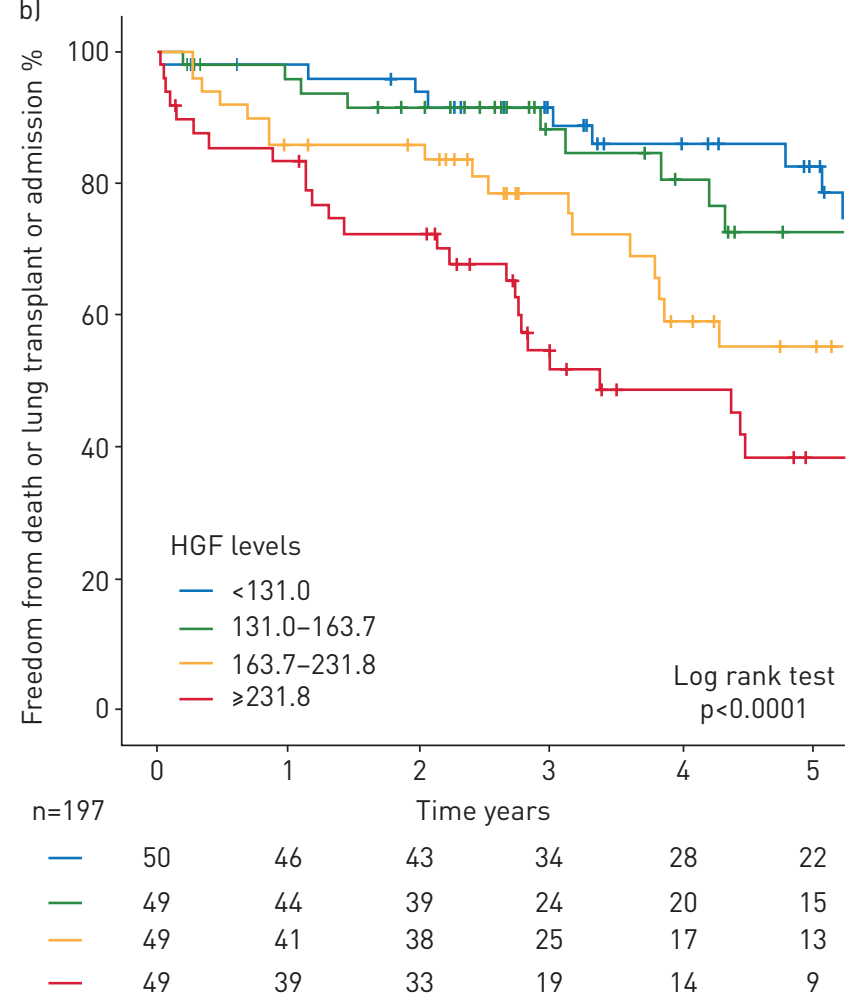

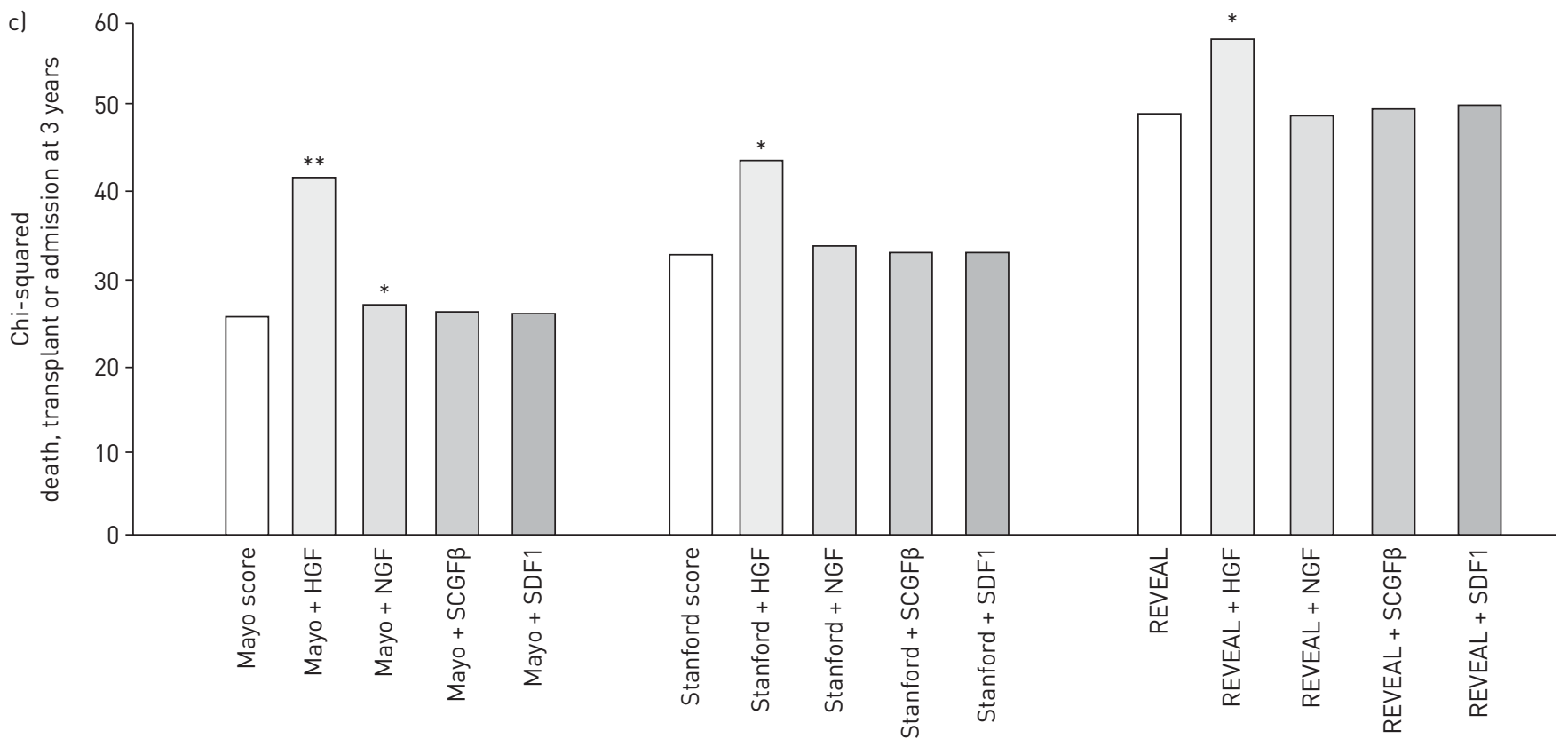

FIGURE 3 Prognostic value of hepatocyte growth factor (HGF) and its incremental value to risk scores for outcome prediction. 5-year KaplanMeier survival curves for al the primary combined end-point of death, lung transplant or hospitalisation for acute right heart failure of the total pulmonary arterial hypertension (PAH) cohort ( $n=197)$ and b) according to quartiles of HGF levels at baseline. c) Chi-squared of scores and HGF, nerve growth factor (NGF), stem cell growth factor (SCGF) $\beta$ and stromal cell-derived factor (SDF)1 for prediction of the primary end-point (death, transplant or admission for right heart failure) at 3 years in the total PAH cohort ( $n=197)$ using Cox regression. Variables were entered in the model using enter mode. The Mayo right heart score was based on the New York Heart Association (NYHA) functional class, N-terminal pro-brain natriuretic peptide (NT-proBNP) and right ventricular longitudinal strain (RVLS). The Stanford right heart score was based on the NYHA class, NT-proBNP and right ventricular end-systolic remodelling index (RVESRI). The REVEAL score was based on the Registry to Evaluate Early and Long-term PAH Disease Management (REVEAL) groups: low, average, moderate high, high and very high. ${ }^{*}: p<0.05 ;{ }^{* *}: p<0.01$. 

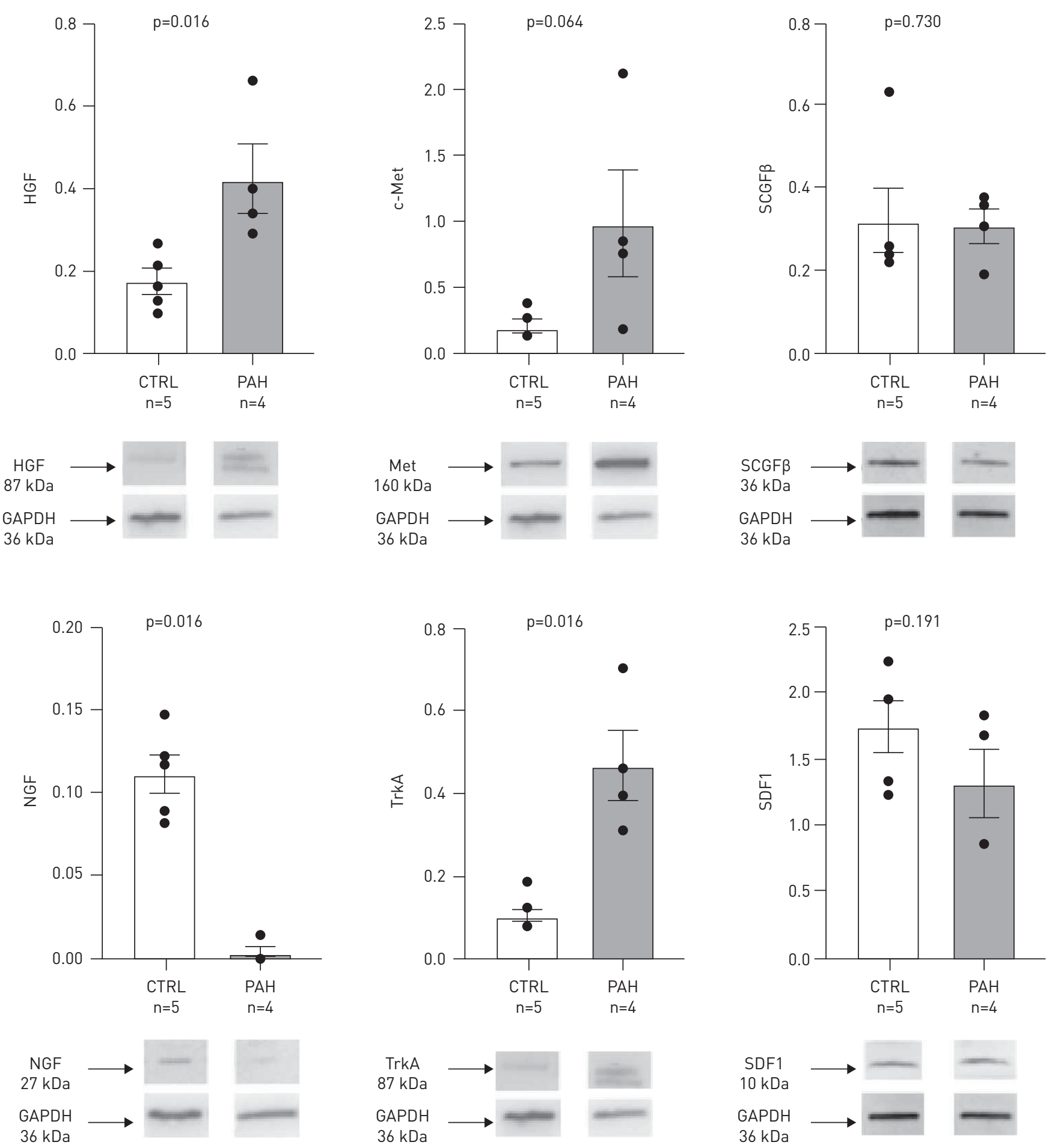

FIGURE 4 Immunoblot of the protein expression of hepatocyte growth factor (HGF), its receptor c-Met, nerve growth factor (NGF) and its receptor TrkA, stem cell growth factor (SCGF) $\beta$ and and stromal cell-derived factor (SDF)1 in the right ventricle of patients with pulmonary arterial hypertension (PAH) and controls (CTRL), and quantification of the signal. Each bar represents the mean \pm SEM signal intensity of Western blots lysate from right ventricular samples from four PAH patients undergoing heart-lung transplant and five control patients (heart donors), which were compared using t-tests. GAPDH: glyceraldehyde-3-phosphate dehydrogenase.

Altered RV expression of growth factors $\mathrm{Hgf}, \mathrm{c}-\mathrm{Met}, \mathrm{Ngf}$ and $\mathrm{Scgf \beta}$ in PAB mice

5 weeks after PAB or sham surgery, the RNA expression of $H g f, c-M e t$ and $\mathrm{Ngf}$ were significantly increased (all $\mathrm{p}<0.01$ ) whereas the expression of $S c g f \beta$ was significantly decreased $(\mathrm{p}<0.001)$ in $\mathrm{RV}$ homogenates from $\mathrm{PAB}$ compared to shams, as presented in supplementary figure E6. 


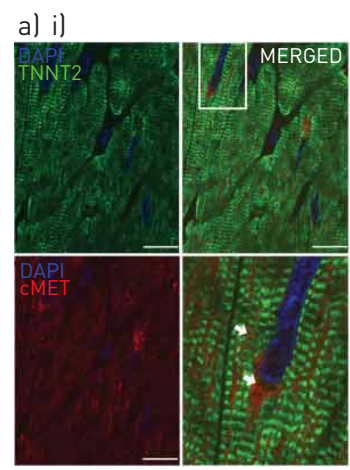

b) i)

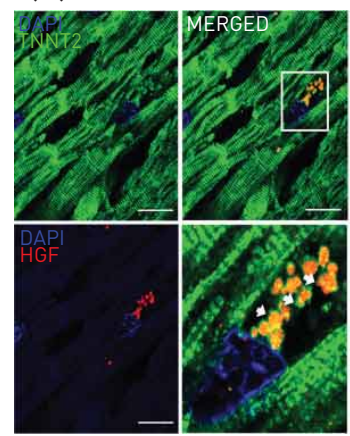

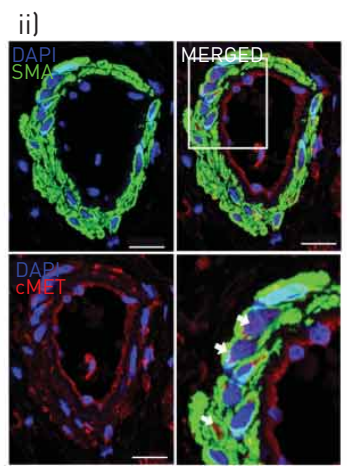

ii)

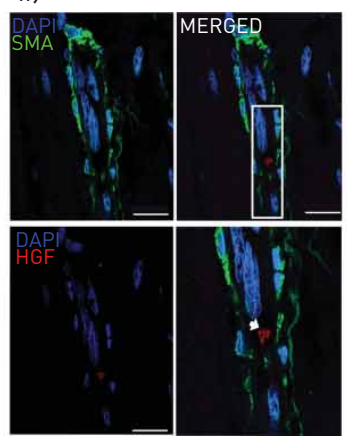

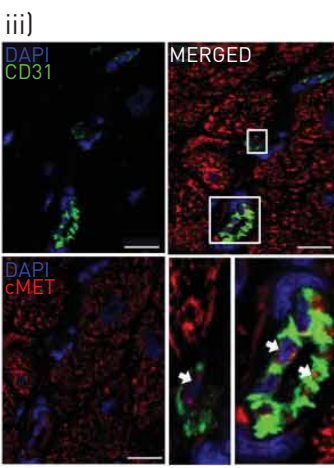

iii)

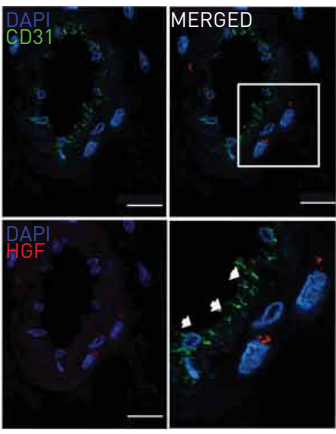

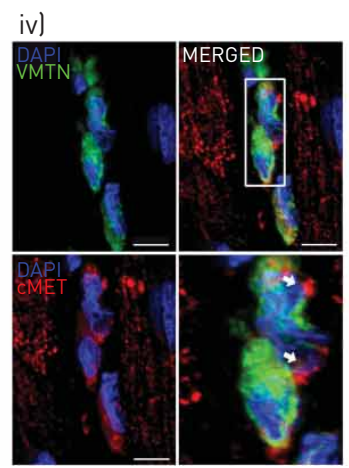
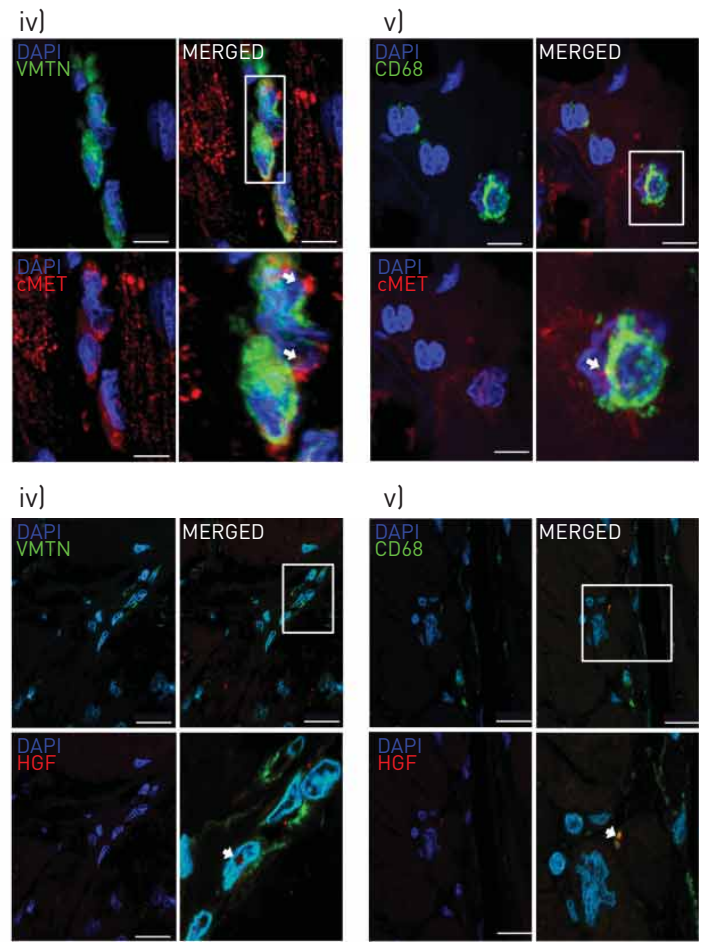

v)

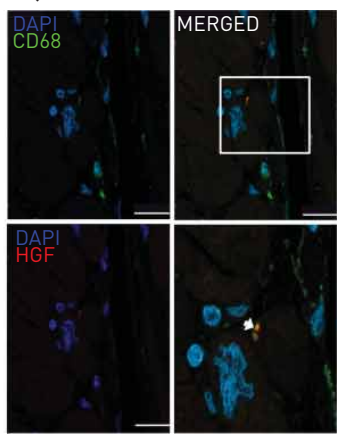

FIGURE 5 Localisation of a) c-Met (red) and b) its ligand hepatocyte growth factor (HGF; red) in right ventricular biopsies from a patient with idiopathic pulmonary arterial hypertension by immunostaining, double-labelled in green with i) TNNT2 troponin (cardiomyocyte), ii) $\alpha$-smooth muscle actin (SMA), iii) CD31 (endothelial cells), iv) vimentin (VMTN) (fibroblast) or v) CD68 (macrophage). No immunoreactivity was seen in cells incubated with the secondary antibody but no primary antibody. Scale bars=10 $\mu \mathrm{m}$. DAPI: 4', 6'-diamidino-2-phenylindole.

\section{Discussion}

This targeted proteomics study identifies four circulating cytokines associated with RHMP, independently from pulmonary haemodynamic severity. HGF emerges as a strong predictor of clinical worsening at 3 years, incremental to the REVEAL score. The protein expression of c-Met and HGF is increased in explanted RVs from patients with PAH who underwent heart-lung transplant in cardiomyocytes, smooth muscle cells and endothelial cells. The PAB mouse model further confirms increased RV gene expression of $\mathrm{Hgf}$ and $c-M e t$, suggesting that PAB is a valid model to further mechanistically study the involvement of this pathway in RV adaptation to $\mathrm{PH}$ over time, and answer the question of whether increasing HGF expression represents a beneficial or deleterious response; information potentially useful from a therapeutic aspect. The study originality resides in its design (going from supervised bedside -omics screening to the target organ and bench analysis), which is a novel approach to the pressure-overloaded right heart. This approach has allowed us to use the blood as a window to right heart failure in PAH, leading to the identification of $\mathrm{HGF} / \mathrm{c}-\mathrm{Met}$ as a potential signalling pathway of interest.

HGF is a mesenchymal-derived pleiotropic cytokine binding to its tyrosine kinase receptor c-Met [13]. The HGF/c-Met signalling pathway promotes angiogenesis and cardiomyocytes survival, inhibits fibrosis in experimental myocardial infarction and left cardiomyopathy models [14, 15] and inhibits vascular permeability and inflammation [16, 17]. Despite the cardioprotective effects of HGF on the left heart, several studies have paradoxically reported the association between high circulating levels of HGF and left ventricular remodelling, dysfunction or adverse outcomes in several pressure-overloaded cardiac diseases (hypertension and aortic stenosis), or advanced ischaemic left heart failure [18-20].

Evidence on the molecular role of HGF/c-Met in the right heart is sparse. High levels of HGF have been reported in small cohorts of patients with scleroderma-associated [21] or idiopathic PAH in whom HGF correlated with mPAP [22]. In another study conducted in patients with idiopathic, familial or associated PAH, HGF levels did not correlate with any clinical markers of disease severity [23]. Our study further demonstrates the association of high plasma HGF levels with RHMP beyond pulmonary disease severity. Including two cohorts differing in terms of demographics, prevalence/incidence repartition and functional severity strengthens the results, suggesting that the biomarkers identified are associated with RV adaptation across PAH aetiology. High HGF levels were strongly associated with outcome in our cohort, adding incremental value to the REVEAL risk score, suggesting the timely role of the HGF/c-Met axis on the 
right heart. None of the four RHMP biomarkers, but, additionally, none of the right heart scores or NT-proBNP levels (validated prognostic markers) at baseline were associated with right heart remodelling improvement at 1 year, irrespective of the therapy initiated, illustrating the distinction between prognostic markers and markers predictive of response to therapy. Future studies should investigate whether changes in biomarkers levels from baseline to 1 year would correlate with changes in right heart remodelling.

The origin of increased HGF plasma levels in PAH remains to be fully elucidated, either resulting from increased production of HGF and/or alterations in its systemic clearance. Increased expression of HGF, SDF1 $\alpha$ and other myeloid-activating factors by primary endothelial cells harvested from human PAH lungs under hypoxic conditions have been reported previously [23]. A cardiac contribution of high circulating HGF levels, in the context of damaged myocardium, cannot be excluded. In the present study, we report the increased protein expression of HGF and c-Met in the RV of patients with PAH with end-stage right heart failure requiring a heart-lung transplant, across PAH aetiology. Another original finding is the localisation of these markers in the RV of patients with $\mathrm{PAH}$, suggesting the pleiotropic effect of HGF/c-Met, as previously demonstrated in the left ventricle [13]. The other markers did not show this increased expression in RV samples from patients with PAH as compared to controls, although limited by the number of samples available, which warrants further investigation.

Animal models bring some insights into the potential origin of circulating HGF levels. Increased HGF plasma levels at 2 weeks have been reported in rats with monocrotaline-induced $\mathrm{PH}$, associated with increase in RV mRNA expression of $H g f$ and $c$-Met at 4 weeks, but not in the liver, suggesting a cardiac rather than hepatic origin of plasma HGF [24]. Our PAB model [11] further demonstrates the increased gene expression of $H g f$ and $c$-Met in RV homogenates from PAB compared to shams (adding to the evidence of increased protein expression in our human samples), suggesting that increased circulating levels of HGF in peripheral blood and in the RVs of patients with PAH could reflect an increased HGF production in the RV. However, these data do not allow the conclusion as to whether increased HGF production in the RV reflects a compensatory attempt of the RV to adapt to an increased afterload or whether it represents rather a harmful response of maladaptation, and further studies using the PAB model will be of high value to clarify the timing and role of the HGF-Met pathways in the pressure-overloaded RV.

The potential beneficial role of HGF as a therapy in PAH has been discussed previously [25]. HGF increases expression of the BMPR2 [26], a pathway mutated in the familial form of PAH, downregulated in nongenetic forms and associated with impaired RV hypertrophy and contractility in small animal models of PH [27, 28]. The activation of BMPR2 is of benefit to PAH and associated RV failure [29], therefore the effects of HGF on BMPR2 could constitute a potential therapy targeting the right heart in PAH. Several animal models (e.g. rats with monocrotaline-induced $\mathrm{PH}$, rabbits with shunt flow-induced $\mathrm{PH})$ have shown that exogenous HGF or $\mathrm{Hgf}$ gene transfection reduced the development of $\mathrm{PH}$, which was associated with less marked right heart hypertrophy and lower inflammatory profiles than shams [30-33]. However, the timely and direct effect of the HGF-c-Met axis activation in the right heart remains to be elucidated. Here the PAB model might be of particular value to explore the dynamic changes in plasma and tissue HGF expression over time and potential reversibility with debanding [11].

NGF promotes cardiomyocyte survival and is the main neurotrophic factor increasing cardiac sympathetic activity $[34,35]$. Higher secretion and endothelial expression of NGF from pulmonary arteries of patients with $\mathrm{PH}$ secondary to COPD than controls without $\mathrm{PH}$ have been reported [36]. However, there was no significant increase in the secretion or lung expression of NGF in patients with idiopathic PAH in this study. NGF was further shown to induce hyperreactivity to vasoconstrictors in rat and human isolated pulmonary arteries, proliferation and migration of human pulmonary arterial smooth muscle cells and endothelial cells, and secretion of the pro-inflammatory cytokines IL-1 $\beta$ and TNF- $\alpha$ [36]. Anti-NGF blocking antibodies reversed $\mathrm{PH}$ in the $\mathrm{PH}$ animal models, decreasing pulmonary vascular remodelling, hyperreactivity and inflammation [36]. Another study in rats with RV hypertrophy secondary to monocrotaline-induced $\mathrm{PH}$ reported the upregulation of NGF associated with deterioration of neuronal cellular function [37]. In humans, NGF has been shown to be downregulated in patients with end-stage left heart failure $[38,39]$, consistent with our immunoblot findings, suggesting a time-variability of this factor in heart failure.

Higher plasmatic concentrations of SCGF $\beta$ have been documented in patients with idiopathic and scleroderma-associated PAH as compared to scleroderma patients without PAH [40]. High circulating SCGF $\beta$ levels have been reported in patients with dilated cardiomyopathy and Chagas disease, without association with outcomes [41]. Finally, increased plasmatic levels of SDF1 $\alpha$, involved in pulmonary artery muscularisation in PAH [42], have been reported in 61 patients with idiopathic PAH as compared to healthy controls; in addition, SDF1 $\alpha$ levels correlated with NT-proBNP and RV function [4]. 


\section{Study limitations}

The first limitation of this study derives from its single-centre design. The fact that the two cohorts were collected and analysed at two different time points, excluding the risk of batch effect in the proteomics analysis, contributes to increasing the confidence in the results. The second limitation derives from the cross-sectional nature of the biomarker analyses, which were only available at baseline. Further studies are needed to investigate whether longitudinal changes in circulating biomarkers would be prognostic or predictive of response to therapy in patients with PAH. The third limitation comes from the absence of RV paraffin-embedded biopsies from controls, which precluded us from assessing whether the increase in HGF protein expression in PAH is observed in all cell types or is specific for a cell type.

In conclusion, high plasma levels of HGF are associated with right heart maladaptive phenotype in PAH and are incremental to the REVEAL score for prediction of outcomes. As both RV HGF-cMet protein and gene expression are increased in $\mathrm{PAH}$, this pathway warrants further exploration as a potential RV-specific therapeutic target. Assessing plasma HGF levels might identify PAH patients at risk of right heart failure and outcomes who warrant closer follow-up and intensified PAH therapy.

Acknowledgements: The authors would like to thank Andrew Hsi (Stanford University, Stanford, CA, USA) for Stanford database management, Lilia Lamrani (Marie Lannelongue Hospital - Paris Sud Paris Saclay University, Paris, France) for her help with clinical data collection, and Jean-Baptiste Michel (Paris VII University, Paris, France) for facilitating the procurement of controls cardiac biopsies. The authors would like to address a special thought to late Catherine Rucker-Martin (from Marie Lannelongue Hospital - Paris Sud Paris Saclay University) who contributed to the immunofluorescence experiments and study design. The authors would like to thank the Vera Moulton Wall Center of Pulmonary Hypertension at Stanford and the Stanford Cardiovascular Institute for their funding support.

Conflict of interest: M. Amsallem has received a 2016 Young Investigator Seed Grant from the Vera Moulton Wall Center at Stanford, a 2019-2020 Stanford Maternal and Child Health Research Institute research seed grant, a research grant from Actelion-Janssen and speaker fees from Bayer. A.J. Sweatt has nothing to disclose. J. Arthur Ataam has nothing to disclose. J. Guihaire has nothing to disclose. F. Lecerf has nothing to disclose. M. Lambert has nothing to disclose. M.R. Ghigna has nothing to disclose. M.K. Ali has nothing to disclose. Y. Mao has nothing to disclose. E. Fadel has nothing to disclose. M. Rabinovitch has nothing to disclose. V. de Jesus Perez has nothing to disclose. E. Spiekerkoetter is funded by Stanford Cardiovascular Institute, National Heart Lung Blood Institute (NHLBI) at the National Institute of Health (NIH) grant R01 HL128734 and Department of Defense grant PR161256. O. Mercier has been supported by a public grant overseen by the French National Research Agency as part of the second Investissement d'Avenir program (ANR-15-RHUS-0002). F. Haddad has received research grants from Actelion-Janssen and Philips. R.T. Zamanian has nothing to disclose.

Support statement: This work was supported by the Stanford Maternal and Child Health Research Institute (2019-2020 Seed Grant), National Institutes of Health - National Institute of Heart, Lung and Blood Institute (Career Development Award, 1K23HL15182), Agence Nationale de la Recherche (grant ANR-15-RHUS-0002) and the Vera Moulton Wall Center at Stanford (Young Investigator Seed Grant). The manuscript content is solely the responsibility of authors and does not necessarily represent the official views of these funding sources. Funding information for this article has been deposited with the Crossref Funder Registry.

\section{References}

1 Vonk Noordegraaf A, Westerhof BE, Westerhof N. The relationship between the right ventricle and its load in pulmonary hypertension. J Am Coll Cardiol 2017; 69: 236-243.

2 Rhodes CJ, Wharton J, Ghataorhe P, et al. Plasma proteome analysis in patients with pulmonary arterial hypertension: an observational cohort study. Lancet Respir Med 2017; 5: 717-726.

3 Sweatt AJ, Hedlin HK, Balasubramanian V, et al. Discovery of distinct immune phenotypes using machine learning in pulmonary arterial hypertension. Circ Res 2019; 124: 904-919.

4 Yang T, Li Z-N, Chen G, et al. Increased levels of plasma CXC-chemokine ligand 10, 12 and 16 are associated with right ventricular function in patients with idiopathic pulmonary arterial hypertension. Heart Lung 2014; 43: 322-327.

5 Sydykov A, Mamazhakypov A, Petrovic A, et al. Inflammatory mediators drive adverse right ventricular remodeling and dysfunction and serve as potential biomarkers. Front Physiol 2018; 9: 609.

6 Fine NM, Chen L, Bastiansen PM, et al. Outcome prediction by quantitative right ventricular function assessment in 575 subjects evaluated for pulmonary hypertension. Circ Cardiovasc Imaging 2013; 6: 711-721.

7 Amsallem M, Sweatt AJ, Aymami MC, et al. Right heart end-systolic remodeling index strongly predicts outcomes in pulmonary arterial hypertension: comparison with validated models. Circ Cardiovasc Imaging 2017; 10: e005771.

8 Breen EJ, Tan W, Khan A. The statistical value of raw fluorescence signal in Luminex xMAP based multiplex immunoassays. Sci Rep 2016; 6: 26996.

9 Benza RL, Miller DP, Gomberg-Maitland M, et al. Predicting survival in pulmonary arterial hypertension: insights from the Registry to Evaluate Early and Long-Term Pulmonary Arterial Hypertension Disease Management (REVEAL). Circulation 2010; 122: 164-172.

10 National Research Council. Guide for the Care and Use of Laboratory Animals. 8th Edn. Washington, DC, The National Academies Press, 2011.

11 Boehm M, Tian X, Mao Y, et al. Delineating the molecular and histological events that govern right ventricular recovery using a novel mouse model of pulmonary artery de-banding. Cardiovasc Res 2020; 116: 1700-1709.

12 Johnson WE, Li C, Rabinovic A. Adjusting batch effects in microarray expression data using empirical Bayes methods. Biostatistics 2007; 8: 118-127. 
13 Gallo S, Sala V, Gatti S, et al. HGF/Met axis in heart function and cardioprotection. Biomedicines 2014; 2: 247-262.

14 Aoki M, Morishita R, Taniyama Y, et al. Angiogenesis induced by hepatocyte growth factor in non-infarcted myocardium and infarcted myocardium: up-regulation of essential transcription factor for angiogenesis, ets. Gene Ther 2000; 7: 417-427.

15 Nakamura T, Mizuno S, Matsumoto K, et al. Myocardial protection from ischemia/reperfusion injury by endogenous and exogenous HGF. J Clin Invest 2000; 106: 1511-1519.

16 Mizuno S, Nakamura T. Prevention of neutrophil extravasation by hepatocyte growth factor leads to attenuations of tubular apoptosis and renal dysfunction in mouse ischemic kidneys. Am J Pathol 2005; 166: 1895-1905.

17 Rutella S, Bonanno G, Procoli A, et al. Hepatocyte growth factor favors monocyte differentiation into regulatory interleukin (IL) $-10^{++} \mathrm{IL}-12^{\text {low/neg }}$ accessory cells with dendritic-cell features. Blood 2006; 108: $218-227$.

18 Rychli K, Richter B, Hohensinner PJ, et al. Hepatocyte growth factor is a strong predictor of mortality in patients with advanced heart failure. Heart 2011; 97: 1158-1163.

19 Kuznetsova T, Haddad F, Knez J, et al. Cytokines profile in hypertensive patients with left ventricular remodeling and dysfunction. J Am Soc Hypertens 2015; 9: 975-984.

20 Kim JB, Kobayashi Y, Kuznetsova T, et al. Cytokines profile of reverse cardiac remodeling following transcatheter aortic valve replacement. Int J Cardiol 2018; 270: 83-88.

21 Riccieri V, Stefanantoni K, Vasile M, et al. Abnormal plasma levels of different angiogenic molecules are associated with different clinical manifestations in patients with systemic sclerosis. Clin Exp Rheumatol 2011; 29: Suppl. 65, S46-S52.

22 Liang M, Pang Y, Zhang S, et al. Utility of hepatocyte growth factor as a biomarker for early diagnosis of pulmonary artery hypertension. Mol Diagn Ther 2016; 20: 463-468.

23 Farha S, Asosingh K, Xu W, et al. Hypoxia-inducible factors in human pulmonary arterial hypertension: a link to the intrinsic myeloid abnormalities. Blood 2011; 117: 3485-3493.

24 Radik M, Kmecova Z, Veteskova J, et al. Hepatocyte growth factor plays a particular role in progression of overall cardiac damage in experimental pulmonary hypertension. Int J Med Sci 2019; 16: 854-863.

25 Guo Y-H, Su L-X, Guo N, et al. Novel therapy for idiopathic pulmonary arterial hypertension: can hepatocyte growth factor be beneficial? J Geriatr Cardiol 2012; 9: 211-212.

26 Ye L, Lewis-Russell JM, Davies G, et al. Hepatocyte growth factor up-regulates the expression of the bone morphogenetic protein (BMP) receptors, BMPR-IB and BMPR-II, in human prostate cancer cells. Int J Oncol 2007; 30: 521-529.

27 Hemnes AR, Brittain EL, Trammell AW, et al. Evidence for right ventricular lipotoxicity in heritable pulmonary arterial hypertension. Am J Respir Crit Care Med 2014; 189: 325-334.

28 Hautefort A, Mendes-Ferreira P, Sabourin J, et al. Bmpr2 mutant rats develop pulmonary and cardiac characteristics of pulmonary arterial hypertension. Circulation 2019; 139: 932-948.

29 Spiekerkoetter E, Tian X, Cai J, et al. FK506 activates BMPR2, rescues endothelial dysfunction, and reverses pulmonary hypertension. J Clin Invest 2013; 123: 3600-3613.

30 Ono M, Sawa Y, Mizuno S, et al. Hepatocyte growth factor suppresses vascular medial hyperplasia and matrix accumulation in advanced pulmonary hypertension of rats. Circulation 2004; 110: 2896-2902.

31 Ono M, Sawa Y, Fukushima N, et al. Gene transfer of hepatocyte growth factor with prostacyclin synthase in severe pulmonary hypertension of rats. Eur J Cardiothorac Surg 2004; 26: 1092-1097.

32 Wang W, Liu K, Zhang F, et al. Recombinant human hepatocyte growth factor transfection alleviates hyperkinetic pulmonary artery hypertension in rabbit models. J Thorac Cardiovasc Surg 2013; 146: 198-205.

33 Chen J, Zhang H, Zhang R, et al. Transfer of human hepatocyte growth factor reduces inflammation and prevents pulmonary arterial remodeling in monocrotaline-induced pulmonary arterial hypertensive rats. Int $J$ Clin Exp Pathol 2014; 7: 8763-8769.

34 Hassankhani A, Steinhelper ME, Soonpaa MH, et al. Overexpression of NGF within the heart of transgenic mice causes hyperinnervation, cardiac enlargement, and hyperplasia of ectopic cells. Dev Biol 1995; 169: 309-321.

35 Caporali A, Sala-Newby GB, Meloni M, et al. Identification of the prosurvival activity of nerve growth factor on cardiac myocytes. Cell Death Differ 2008; 15: 299-311.

36 Freund-Michel V, Cardoso Dos Santos M, Guignabert C, et al. Role of nerve growth factor in development and persistence of experimental pulmonary hypertension. Am J Respir Crit Care Med 2015; 192: 342-355.

37 Kimura K, Ieda M, Kanazawa H, et al. Cardiac sympathetic rejuvenation: a link between nerve function and cardiac hypertrophy. Circ Res 2007; 100: 1755-1764.

38 Kaye DM, Vaddadi G, Gruskin SL, et al. Reduced myocardial nerve growth factor expression in human and experimental heart failure. Circ Res 2000; 86: E80-E84.

39 Qin F, Vulapalli RS, Stevens SY, et al. Loss of cardiac sympathetic neurotransmitters in heart failure and NE infusion is associated with reduced NGF. Am J Physiol Heart Circ Physiol 2002; 282: H363-H371.

40 Stefanantoni K, Sciarra I, Vasile M, et al. Elevated serum levels of macrophage migration inhibitory factor and stem cell growth factor $\beta$ in patients with idiopathic and systemic sclerosis associated pulmonary arterial hypertension. Reumatismo 2015; 66: 270-276.

41 Wang Y, Khan A, Heringer-Walther S, et al. Prognostic value of circulating levels of stem cell growth factor beta (SCGF beta) in patients with Chagas' disease and idiopathic dilated cardiomyopathy. Cytokine 2013; 61: 728-731.

42 Yuan K, Liu Y, Zhang Y, et al. Mural cell SDF1 signaling is associated with the pathogenesis of pulmonary arterial hypertension. Am J Respir Cell Mol Biol 2020; 62: 747-759. 